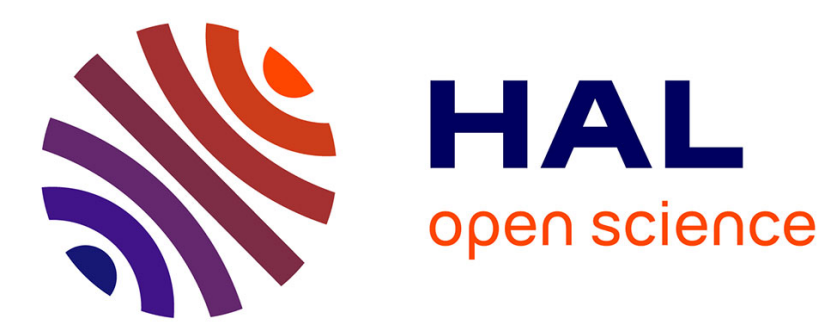

\title{
ON EQUIVALENCE OF SUPER LOG SOBOLEV AND NASH TYPE INEQUALITIES
}

\author{
Marco Biroli, Patrick Maheux
}

\section{To cite this version:}

Marco Biroli, Patrick Maheux. ON EQUIVALENCE OF SUPER LOG SOBOLEV AND NASH TYPE INEQUALITIES. Colloquium Mathematicum, 2014, 137 (2), pp.189-208. 10.4064/cm137-2-4 . hal$00465177 \mathrm{v} 3$

\section{HAL Id: hal-00465177 \\ https://hal.science/hal-00465177v3}

Submitted on 20 Nov 2014

HAL is a multi-disciplinary open access archive for the deposit and dissemination of scientific research documents, whether they are published or not. The documents may come from teaching and research institutions in France or abroad, or from public or private research centers.
L'archive ouverte pluridisciplinaire HAL, est destinée au dépôt et à la diffusion de documents scientifiques de niveau recherche, publiés ou non, émanant des établissements d'enseignement et de recherche français ou étrangers, des laboratoires publics ou privés. 


\title{
ON EQUIVALENCE OF SUPER LOG SOBOLEV AND NASH TYPE INEQUALITIES
}

\author{
MARCO BIROLI AND PATRICK MAHEUX
}

\begin{abstract}
We prove the equivalence of Nash type and super log Sobolev inequalities for Dirichlet forms. We also show that both inequalities are equivalent to Orlicz-Sobolev type inequalities. No ultracontractivity of the semigroup is assumed. It is known that there is no equivalence between super log Sobolev or Nash type inequalities and ultracontractivity. We discuss Davies-Simon's counterexample as borderline case of this equivalence and related open problems.
\end{abstract}

\section{INTRODUCTION}

Let $\left(T_{t}\right)_{t>0}=\left(e^{-A t}\right)_{t>0}$ be a symmetric submarkovian semigroup with infinitesimal generator $-A$ on $L^{2}(X, \mu)$ where $(X, \mu)$ is a $\sigma$-finite measure space. The symmetry reads as

$$
\left(T_{t} f, g\right)=\left(f, T_{t} g\right), \quad f, g \in L^{2}, \quad t>0,
$$

and the submarkovian property reads as

$$
0 \leq f \leq 1 \Rightarrow 0 \leq T_{t} f \leq 1, \quad f \in L^{2} .
$$

Moreover, the semigroup $\left(T_{t}\right)_{t>0}$ is a $C_{0}$-contraction semigroup on $L^{2}$ which extends to a $C_{0}$-contraction semigroup $T_{t}:=T_{t}^{(p)}$ on each $L^{p}=L^{p}(X, \mu)$ with $1 \leq p<+\infty$ and acts as a contraction on $L^{\infty}$. The infinitesimal generator $-A$ is defined by

$$
A f:=\lim _{t \rightarrow 0^{+}} \frac{f-T_{t} f}{t},
$$

for $f \in \mathcal{D}(A)$ i.e. $f \in L^{2}$ such that $\lim _{t \rightarrow 0^{+}} \frac{f-T_{t} f}{t}$ exists in $L^{2}$. In particular, the operator $A$ is non-negative and self-adjoint on $L^{2}$.

The associated Dirichlet form $\mathcal{E}$ is defined as follows. Let $\mathcal{D}(\mathcal{E})=\mathcal{D}(\sqrt{A})$ where $\sqrt{A}$ is the positive square root of $A$. We set $\mathcal{E}(f, g)=(\sqrt{A} f, \sqrt{A} g)$ for $f, g \in \mathcal{D}(\mathcal{E})$. Then the bilinear form $\mathcal{E}$ is a positive, symmetric, bilinear,

2010 Mathematics Subject Classification. Primary 39B62.

Key words and phrases. Ultracontractivity, super log Sobolev inequality, Nash type inequality, Orlicz-Sobolev inequality, semigroups of operators, Dirichlet form, heat kernel, infinite dimensional torus. 
closed, densely defined form on $L^{2}(\mu)$. Moreover, the form $\mathcal{E}$ satisfies the following contraction property:

$$
\forall f \in \mathcal{D}(\mathcal{E}), g=(f \wedge 1) \vee 0 \Longrightarrow g \in \mathcal{D}(\mathcal{E}) \text { and } \mathcal{E}(g) \leq \mathcal{E}(f)
$$

where $\mathcal{E}(f):=\mathcal{E}(f, f)$. Furthermore, there is a bijective correspondence between Dirichlet forms and submarkovian symmetric semigroups, see $[\mathrm{F}]$.

A fundamental property of semigroups of operators $\left(T_{t}\right)_{t>0}$ is ultracontractivity, that is the existence of a non-increasing function $a$ from $(0,+\infty)$ to itself such that for any $f \in L^{1}$,

$$
\left\|T_{t} f\right\|_{\infty} \leq a(t)\|f\|_{1}, \quad t>0 .
$$

In that case, we will say that the semigroup is ultracontractive.

Under some conditions described for instance in [GH, Section 3.3] (see also [W2, Proposition 3.3.11]), ultracontractivity of a semigroup (1.2) implies the existence of a heat kernel $h_{t}(x, y)$ for this semigroup

$$
T_{t} f(x)=\int_{X} h_{t}(x, y) f(y) d \mu(y),
$$

and uniform bounds on this kernel

$$
\sup _{x, y \in X} h_{t}(x, y) \leq a(t), \quad t>0 .
$$

Conversely, the existence of a heat kernel and uniform bounds obviously implies ultracontractivity of the semigroup.

Before going further, let us discuss some equivalent formulation of ultracontractivity. By interpolation, semigroup property, duality and symmetry, inequality (1.2) is equivalent to the existence of a non-increasing function $c:(0,+\infty) \longrightarrow(0,+\infty)$ such that for any $f \in L^{1}$,

$$
\left\|T_{t} f\right\|_{2} \leq c(t)\|f\|_{1}, \quad t>0
$$

or equivalently, for any $f \in L^{2}$,

$$
\left\|T_{t} f\right\|_{\infty} \leq c(t)\|f\|_{2}, \quad t>0 .
$$

More precisely, (1.2) implies (1.3) and (1.4) with $c(t) \leq \sqrt{a(t)}$ and conversely (1.3) or (1.4) implies (1.2) with $a(t) \leq c^{2}(t / 2)$, see [D].

It is known that some regularization properties (for instance ultracontractivity) of the semigroup $\left(T_{t}\right)_{t>0}$ can be quantified by functional inequalities satisfied by the infinitesimal generator $-A$. Let us recall two fundamental results in that direction.

Let $M:(0,+\infty) \rightarrow \mathbb{R}$ be a function. For any $y \in \mathbb{R}$, we set

$$
\Lambda(y)=\sup _{t>0}\{t y-2 t M(1 / 2 t)\} \in(-\infty,+\infty] .
$$


Note that the function $\Lambda$ is the Legendre transform of $t \mapsto 2 t M(1 / 2 t)$.

In [C, Proposition II.2], T. Coulhon proved that if $\left(T_{t}\right)_{t>0}$ is ultracontractive with $c(t)=e^{M(t)}$ in (1.3). Then the following Nash type inequality

$$
\Theta\left(\|f\|_{2}^{2}\right) \leq \mathcal{E}(f), \quad f \in \mathcal{D}(\mathcal{E}),\|f\|_{1} \leq 1,
$$

holds true with $\Theta(x)=x \Lambda(\log x), x>0$ and $\Lambda$ given by (1.5). This specific form of $\Theta$ will be of importance for the formulation of our main result Theorem 1.1 as already noticed in $[\mathrm{BM}]$ in a particular case. This inequality can be seen as a weak form of Sobolev inequality, see for instance $[\mathrm{C}]$, [BCLS], [VSC], [W2], [BGL].

On the other hand, E.B Davies and B. Simon proved in $[D$, Theorem 2.2.3] that if $\left(T_{t}\right)_{t>0}$ is ultracontractive with $c(t)=e^{\beta(t)}$ in (1.3). Then for any non-negative function $f$ in $\mathcal{D}(\mathcal{E}) \cap L^{1} \cap L^{\infty}$, it implies $f^{2} \log f \in L^{1}$ and the following super log Sobolev inequality (1.7) holds true

$$
\int_{X} f^{2} \log f d \mu \leq t \mathcal{E}(f)+\beta(t)\|f\|_{2}^{2}+\|f\|_{2}^{2} \log \|f\|_{2}, \quad t>0 .
$$

This inequality is modeled on the celebrated Gross' inequality [G1] and equivalent to supercontractivity of the semigroup $\left(T_{t}\right)_{t>0}$ i.e. for each $t>0$, $T_{t}$ is bounded from $L^{2}$ to $L^{4}$, see [G2, Theorem 3.7].

T. Coulhon's and Davies-Simon's results assert that ultracontractivity of the semigroup implies Nash type inequality (1.6) and super log Sobolev inequality (1.7) respectively for the generator of the semigroup. Then it is natural to ask whether there exist direct relationships between Nash type and super log Sobolev inequalities without ultracontractivity assumption.

Our main theorem provides a positive answer to that question.

Main Theorem 1.1. Let $\mathcal{E}$ be a Dirichlet form with domain $\mathcal{D}(\mathcal{E})$. Then the following statements are equivalent

(1) There exists a function $M_{1}:(0,+\infty) \rightarrow \mathbb{R}$ such that for any $f \in$ $\mathcal{D}(\mathcal{E})$

$$
\int_{X} f^{2} \log \left(\frac{|f|}{\|f\|_{2}}\right) d \mu \leq t \mathcal{E}(f)+M_{1}(t)\|f\|_{2}^{2}, \quad t>0 .
$$

(2) There exits a function $M_{2}:(0,+\infty) \rightarrow \mathbb{R}$ such that, for any $f \in$ $\mathcal{D}(\mathcal{E}) \cap L^{1}$ with $\|f\|_{1} \leq 1$,

$$
\|f\|_{2}^{2} \Lambda\left(\log \|f\|_{2}^{2}\right) \leq \mathcal{E}(f)
$$

where $\Lambda$ is defined by (1.5) with $M=M_{2}$. 
(3) There exists a function $M_{3}:(0,+\infty) \rightarrow \mathbb{R}$ and constants $c_{1}, c_{2}>0$ such that, for any $f \in \mathcal{D}(\mathcal{E}) \cap L^{1}$ with $\|f\|_{2}^{2}=1$,

$$
c_{1} \int_{X} f^{2} \Lambda_{+}\left(\log c_{2}^{2} f^{2}\right) d \mu \leq \mathcal{E}(f)
$$

with $\Lambda_{+}=\sup (\Lambda, 0)$ where $\Lambda$ is defined by (1.5) with $M=M_{3}$.

The proof is given in Section 2.

Remark 1.2. (1) Super log Sobolev inequality (1.8) implies Nash type inequality (1.9) with $M=M_{2}=M_{1}$ in the definition (1.5) of $\Lambda$.

(2) Nash type inequality (1.9) implies Orlicz-Sobolev inequality (1.10) with $M_{3}=M_{2}, c_{1}=16^{-1}, c_{2}=8^{-1}$.

(3) Orlicz-Sobolev inequality (1.10) implies super log Sobolev inequality (1.8) with $M_{1}(t)=M_{3}\left(c_{3} t\right)+c_{4}$ and $c_{3}=c_{1}, c_{4}=-\log c_{2}$.

(4) We do not need to assume that $\mathcal{E}$ is a Dirichlet form for the implications $(1.8) \Longrightarrow(1.9)$ and $(1.10) \Longrightarrow(1.8)$. But the assumption that $\mathcal{E}$ is a Dirichlet form is fundamental for the implication $(1.9) \Longrightarrow$ (1.10).

(5) Inequalities similar to (1.10) called F-Sobolev inequality have been obtained by F.Y. Wang under a super Poincaré inequality assumption, see [W1], [W2]. See also the comments at the end of Section 3.2 .

As a direct consequence of Theorem 1.1, we can provide an alternative proof of Nash type inequality (1.6) under an ultracontractivity assumption. Indeed, we just need to apply successively Davies-Simon's result to deduce super log Sobolev inequality (1.7) and Theorem 1.1 to get exactly Nash type inequality (1.6). Likewise, we can provide an alternative proof of super log Sobolev inequality (1.7) (with some loss on $\beta$ ) under an ultracontractivity assumption. Here again, we just need to apply successively Coulhon's result to deduce Nash type inequality (1.6) and Theorem 1.1 to get super log Sobolev inequality (1.7).

In practice, functional inequalities are used to prove ultracontractivity and, consequently, to get bounds on the heat kernel of the semigroup, for instance, under a Nash type inequality or a super log Sobolev inequality assumption. Here, we briefly discuss these aspects of the theory by first quoting one of the results of T. Coulhon under Nash type inequalities assumption. We restrict the statement to the class of submarkovian semigroups. For a more general statement, we refer to Proposition II.1 of $[\mathrm{C}]$. Assume that a quadratic form $\mathcal{E}(f)=(A f, f)$ satisfies (1.6) for a continuous function 
$\Theta:[0,+\infty) \rightarrow[0,+\infty)$ such that $\frac{1}{\Theta}$ is integrable at infinity. Then the semigroup $\left(T_{t}\right)_{t>0}$ is ultracontractive and satisfies

$$
\left\|T_{t} f\right\|_{\infty} \leq m(t)\|f\|_{1}, \quad 0<t<t_{0}
$$

where $m$ is the inverse function of $y \mapsto p(y)=\int_{y}^{\infty} \frac{d x}{\Theta(x)}, y>0$ and $t_{0}=$ $\frac{1}{2} \int_{0}^{+\infty} \frac{d x}{\Theta(x)} \in(0,+\infty]$, see also Theorem 7.4.5 in [BGL] for a variant.

On the other hand, Theorem 2.2.7 and its Corollary 2.2.8 in [D], which assume a super log Sobolev inequality, allows us to deduce ultracontractivity under additional conditions on $\beta$ in (1.7). The method have been refined later by D. Bakry in [B], see also Theorem 7.1.2 in [BGL]. For this latter method, we will not go further in details. Indeed, at the level of functional inequalities Theorem 1.1 says that super log Sobolev inequalities imply Nash type inequalities (in fact equivalent) with exactly the same formula (1.6) for $\Theta$ as in T. Coulhon's result. So, we will emphasize this first method to get ultracontractive bounds for various classes of behaviour in Section 3 . We refer to $[\mathrm{B}],[\mathrm{BGL}],[\mathrm{C}],[\mathrm{D}],[\mathrm{DS}],[\mathrm{W} 2]$ (and references therein) for more details on the subject of ultracontractivity. Note that the sharp Euclidean ultracontractive bounds (heat kernel bounds) may be deduced from the sharp super log Sobolev inequality in $\mathbb{R}^{n}$, see [BCL], [B], and also [BGL, Corollary 7.1.6] for a precise statement.

In Section 3, our goal is to describe briefly some classes of ultracontractivity and their pertaining functional inequalities using Theorem 1.1 and known results as $(1.6),(1.7),(1.11)$. In particular, we examine two classes where these properties are equivalent. We also exhibit a class of ultracontractivity where the equivalence does not hold. In Section 4, we provide examples for these three particular classes of ultracontractivity. The non equivalence will be confirmed by the counterexample of E.B Davies and B. Simon described in Section 4.3. This leads us to discuss open problems in Section 5 .

The paper is organized as follows.

In Section 2, we prove our main result Theorem 1.1. In Section 3, we exhibit the functional inequalities for the polynomial, the one and the doubleexponential classes of ultracontractivity. In Section 4, we briefly describe some examples belonging to these classes and the Davies-Simon's counterexample. In Section 5, we suggest some open problems about the doubleexponential class and beyond.

\section{Proof of Theorem 1.1}


2.1. Super log Sobolev inequality implies Nash type inequality. We prove that $\log$ Sobolev inequality (1.8) implies Nash type inequality (1.9) with $M_{2}=M_{1}$ using the following convexity argument.

Lemma 2.1. If $f \in L^{1} \cap L^{2}$ with $f \geq 0$ and $\|f\|_{1} \leq 1$ then

$$
\|f\|_{2}^{2} \log \|f\|_{2} \leq \int_{X} f^{2} \log \left(|f| /\|f\|_{2}\right) d \mu .
$$

Proof. First, assume $\|f\|_{1}=1$. We deduce (2.1) by applying Jensen's inequality to the convex function $\Psi(x)=x \log x$ and the probability measure $d \nu=|f| d \mu$. For any $f \in L^{1} \cap L^{2}$ with $f$ non identically zero, we get by homogeneity,

$$
\|f\|_{2}^{2} \log \left(\|f\|_{2} /\|f\|_{1}\right) \leq \int_{X} f^{2} \log \left(|f| /\|f\|_{2}\right) d \mu
$$

or equivalently,

$$
\|f\|_{2}^{2} \log \|f\|_{2} \leq \int_{X} f^{2} \log \left(|f| /\|f\|_{2}\right) d \mu+\|f\|_{2}^{2} \log \|f\|_{1} .
$$

If we assume $\|f\|_{1} \leq 1$ then $\log \|f\|_{1} \leq 0$ and we get immediately (2.1). The proof of the lemma is complete.

Now, we assume that super log Sobolev inequality (1.8) holds true. By Lemma 2.1, we deduce that for any function $f$ in $\mathcal{D}(\mathcal{E})$ with $\|f\|_{1} \leq 1$,

$$
\|f\|_{2}^{2} \log \|f\|_{2} \leq s \mathcal{E}(f)+M_{1}(s)\|f\|_{2}^{2}, \quad s>0 .
$$

Hence, for any $s>0$,

$$
\|f\|_{2}^{2}\left(\frac{1}{2 s} \log \|f\|_{2}^{2}-\frac{1}{s} M_{1}(s)\right) \leq \mathcal{E}(f) .
$$

By taking the supremum over $s>0$, it yields

$$
\|f\|_{2}^{2} \Lambda\left(\log \|f\|_{2}^{2}\right) \leq \mathcal{E}(f)
$$

where $\Lambda$ is defined by (1.5) with $M=M_{1}$. Thus Nash type inequality (1.9) and (1) of Remark 1.2 are proved. This finishes the proof.

Remark 2.2. Note that we do not use any assumption on the functional $\mathcal{E}$ in this proof. Moreover, the function $\Lambda$ is automatically finite on the set $\left\{\log \|f\|_{2}^{2}: f \in \mathcal{D}(\mathcal{E}) \cap L^{1},\|f\|_{1} \leq 1\right\}$.

\subsection{Nash type inequality implies Orlicz-Sobolev type inequality.}

We use the cut-off method developed in [BCLS] to show that Nash type inequality (1.9) implies Orlicz-Sobolev type inequality (1.10). The fact that $\mathcal{E}$ is a Dirichlet form is fundamental due to the next lemma. 
Lemma 2.3. Let $\mathcal{E}$ be a Dirichlet form with domain $\mathcal{D}(\mathcal{E})$. Let $f$ be any non-negative function in $\mathcal{D}(\mathcal{E})$. We set for any $\rho>1$ and any $k \in \mathbb{Z}$,

$$
f_{\rho, k}=\left(f-\rho^{k}\right)^{+} \wedge \rho^{k}(\rho-1) .
$$

Then $f_{\rho, k} \in \mathcal{D}(\mathcal{E})$ and

$$
\sum_{k \in \mathbb{Z}} \mathcal{E}\left(f_{\rho, k}\right) \leq \mathcal{E}(f)
$$

This lemma can be compared with Corollary 2.3 of [BCLS] and Lemma 3.3.2 of [W2]. In what follows, we will denote indifferently $\mathcal{E}(f)$ or $\mathcal{E}(f, f)$. The starting point is the following important remark ${ }^{1}$.

Remark 2.4. Let $\lambda \geq 0$. Let $g \in \mathcal{D}(\mathcal{E})$ with $g \geq 0$. Denote the support of $g$ by $\operatorname{supp}(g)=\{x \in X: g(x) \neq 0\}$. If $h \in \mathcal{D}(\mathcal{E})$ with $0 \leq h \leq \lambda$, satisfies $h=\lambda$ on $\operatorname{supp}(g)$, then $\mathcal{E}(h, g) \geq 0$, see [A, (ii) p.2].

We prove the remark as follows. For any $\varepsilon>0$, we have $(h+\varepsilon g) \wedge \lambda=h$. By Dirichlet property

$$
\mathcal{E}(h)=\mathcal{E}((h+\varepsilon g) \wedge \lambda) \leq \mathcal{E}(h+\varepsilon g)=\mathcal{E}(h)+2 \varepsilon \mathcal{E}(h, g)+\varepsilon^{2} \mathcal{E}(g) .
$$

Subtracting $\mathcal{E}(h)$ on both sides of this inequality and dividing by $\varepsilon>0$, it yields

$$
0 \leq 2 \mathcal{E}(h, g)+\varepsilon \mathcal{E}(g) .
$$

When $\varepsilon$ goes to 0 , we get $\mathcal{E}(h, g) \geq 0$ as stated.

Proof. (Lemma 2.3). Let $f \in \mathcal{D}(\mathcal{E})$ be non-negative. Fix $\rho>0$. For any $n \in \mathbb{N}$, we set $f_{\rho}^{n}=f \wedge \rho^{n+1}=\rho^{n+1}\left(\left(\frac{f}{\rho^{n+1}} \wedge 1\right) \vee 0\right)$. By Dirichlet property, $f_{\rho}^{n}, f_{\rho}^{-(n+1)}$ and $f_{\rho, k}$ belong to $\mathcal{D}(\mathcal{E})$. Indeed, each one of these expressions are normal contractions of $f$ of the form $\phi(f)$ with $\phi(u)=((u-a) \vee 0) \wedge b$ where $a, b>0$, see [F, p.5].

We apply Remark 2.4 to $h=f_{\rho}^{-(n+1)}, \lambda=\rho^{-n}$ and $g=f_{\rho, k}$ with $k=-n, \cdots, n$. We deduce $\mathcal{E}\left(f_{\rho, k}, f_{\rho}^{-(n+1)}\right) \geq 0$ for $k=-n, \cdots, n$.

Let $(p, k) \in \mathbb{Z}^{2}$ with $p<k$. Once again, we apply Remark 2.4 with $h=f_{\rho, p}, \lambda=\rho^{p}(\rho-1)$ and $g=f_{\rho, k}$, it yields $\mathcal{E}\left(f_{\rho, p}, f_{\rho, k}\right) \geq 0$. By the relation

$$
f_{\rho}^{n}=\sum_{k=-\infty}^{n} f_{\rho, k}=\sum_{k=-n}^{n} f_{\rho, k}+f_{\rho}^{-(n+1)}
$$

and the Dirichlet property, we obtain

$$
\mathcal{E}(f) \geq \mathcal{E}\left(f_{\rho}^{n}\right)=\mathcal{E}\left(\sum_{k=-n}^{n} f_{\rho, k}\right)+2 \sum_{k=-n}^{n} \mathcal{E}\left(f_{\rho, k}, f_{\rho}^{-(n+1)}\right)+\mathcal{E}\left(f_{\rho}^{-(n+1)}\right) .
$$

\footnotetext{
${ }^{1}$ The main argument was pointed out to us by G.Allain, see $[A, p .2,5]$.
} 
Since the two last terms are non-negative and by developing the third term, we get

$$
\mathcal{E}(f) \geq \sum_{k, p=-n}^{n} \mathcal{E}\left(f_{\rho, p}, f_{\rho, k}\right)=\sum_{k=-n}^{n} \mathcal{E}\left(f_{\rho, k}, f_{\rho, k}\right)+\sum_{k \neq p, k, p \geq-n}^{n} \mathcal{E}\left(f_{\rho, p}, f_{\rho, k}\right) .
$$

Since the last term of the right-hand side is non-negative, we arrive at

$$
\mathcal{E}(f) \geq \sum_{k=-n}^{n} \mathcal{E}\left(f_{\rho, k}, f_{\rho, k}\right) .
$$

The lemma is proved by passing to the limit as $n \rightarrow+\infty$.

We are now in position to prove Orlicz-Sobolev inequality (1.10). We divide the proof into two steps.

Step 1. Let $f \in L^{2}$ be a non-negative function such that $\|f\|_{2}=1$. Let $k \in \mathbb{Z}$. We define $f_{k}:=f_{2, k}=\left(f-2^{k}\right)^{+} \wedge 2^{k}$. By Hölder's inequality,

$$
\left\|f_{k}\right\|_{1}^{2} \leq\left\|f_{k}\right\|_{2}^{2} \mu\left(f \geq 2^{k}\right) .
$$

On the other hand, by Bienaymé-Chebyshev's inequality we have

$$
2^{2(k-1)} \mu\left(f \geq 2^{k}\right) \leq\left\|f_{k-1}\right\|_{2}^{2} \leq\|f\|_{2}^{2} \leq 1
$$

Combining inequalities (2.6) and (2.7), we obtain

$$
2^{k-1} \leq\left\|f_{k}\right\|_{2} /\left\|f_{k}\right\|_{1} .
$$

Again by Bienaymé-Chebyshev's inequality, we have

$$
2^{2 k} \mu\left(f \geq 2^{k+1}\right) \leq\left\|f_{k}\right\|_{2}^{2} .
$$

Step 2. Assume that Nash type inequality (1.9) holds true. For convenience set $B(x)=\Lambda\left(\log x^{2}\right)$. For a function $H$ defined on $[0,+\infty)$, we define $H_{+}$its non-negative part by $H_{+}(x)=\max (H(x), 0), x \geq 0$. As the quadratic form $\mathcal{E}$ is non-negative, we have for any $g \in \mathcal{D}(\mathcal{E}) \cap L^{1}$ with $\|g\|_{1}=1$,

$$
\|g\|_{2}^{2} \Lambda_{+}\left(\log \|g\|_{2}^{2}\right)=\|g\|_{2}^{2} B_{+}\left(\|g\|_{2}\right) \leq \mathcal{E}(g) .
$$

By homogeneity, it implies for any $g \in \mathcal{D}(\mathcal{E}) \cap L^{1}$ and $g \neq 0$,

$$
\|g\|_{2}^{2} \Lambda_{+}\left(\log \|g\|_{2}^{2} /\|g\|_{1}^{2}\right)=\|g\|_{2}^{2} B_{+}\left(\|g\|_{2} /\|g\|_{1}\right) \leq \mathcal{E}(g) .
$$

Let $f$ and $f_{k}$ be as in Lemma 2.3. We apply inequality (2.10) to $g=f_{k}$, it yields

$$
\left\|f_{k}\right\|_{2}^{2} B_{+}\left(\left\|f_{k}\right\|_{2} /\left\|f_{k}\right\|_{1}\right) \leq \mathcal{E}\left(f_{k}\right)
$$

Since $B_{+}$is a non-negative non-decreasing function, we obtain from (2.8) and (2.9),

$$
2^{2 k} B_{+}\left(2^{k-1}\right) \mu\left(f \geq 2^{k+1}\right) \leq \mathcal{E}\left(f_{k}\right), \quad k \in \mathbb{Z},
$$


for any non-negative function $f \in \mathcal{D}(\mathcal{E}) \cap L^{1}$. Let $\lambda>0$ to be chosen later. We discretize the following integral

$$
\begin{aligned}
\int_{X} f^{2} B_{+}(\lambda f) d \mu & =\sum_{k \in \mathbb{Z}} \int_{\left\{2^{k} \leq f<2^{k+1}\right\}} f^{2} B_{+}(\lambda f) d \mu \\
& \leq \sum_{k \in \mathbb{Z}} 2^{2(k+1)} B_{+}\left(\lambda 2^{k+1}\right) \mu\left(f \geq 2^{k}\right) \\
& \leq \sum_{k \in \mathbb{Z}} 2^{2(k+2)} B_{+}\left(\lambda 2^{k+2}\right) \mu\left(f \geq 2^{k+1}\right) .
\end{aligned}
$$

We choose $\lambda=2^{-3}$ and we get

$$
\int_{X} f^{2} B_{+}(f / 8) d \mu \leq 2^{4} \sum_{k \in \mathbb{Z}} 2^{2 k} B_{+}\left(2^{k-1}\right) \mu\left(f \geq 2^{k+1}\right) .
$$

By applying (2.11) and Lemma 2.3 with $\rho=2$, it leads to

$$
\int_{X} f^{2} B_{+}(f / 8) d \mu \leq 2^{4} \sum_{k \in \mathbb{Z}} \mathcal{E}\left(f_{k}\right) \leq 2^{4} \mathcal{E}(f) .
$$

So, Orlicz-Sobolev type inequality (1.10) is proved for $f \geq 0,\|f\|_{2}=1$ with $c_{1}=1 / 16$ and $c_{2}=1 / 8$. For real-valued functions $f \in \mathcal{D}(\mathcal{E})$, we obtain the same conclusion by using $\mathcal{E}(|f|) \leq \mathcal{E}(f)$ which is a consequence of the Dirichlet property. This completes the proof of Orlicz-Sobolev inequality (1.10) and (2) of Remark 1.2.

\subsection{Orlicz-Sobolev type inequalities implies super log Sobolev in-} equalities. In this section, we plan to prove super log Sobolev inequality (1.8) under the assumption of Orlicz-Sobolev type inequality (1.10).

From the definition (1.5) of $\Lambda$, we have for any $t>0$ and $y \geq 0$,

$$
t y / 2-t M_{3}(1 / t) \leq \Lambda(y) \leq \Lambda_{+}(y) .
$$

By the change of variable $t=\left(c_{1} s\right)^{-1}$ for $s>0$ with $c_{1}>0$ as in (1.10), it yields

$$
y / 2 \leq c_{1} s \Lambda_{+}(y)+M_{3}\left(c_{1} s\right) .
$$

Let $f \in \mathcal{D}(\mathcal{E})$. Set $y=\log \left(c_{2}^{2} f^{2}\right)$ with $c_{2}>0$ as in (1.10). Hence

$$
\log \left(c_{2}|f|\right) \leq c_{1} s \Lambda_{+}\left(\log \left(c_{2}^{2} f^{2}\right)\right)+M_{3}\left(c_{1} s\right) .
$$

Multiplying this expression by $f^{2}$ and integrating on $X$ with respect to $\mu$, it leads us to

$$
\begin{aligned}
\int_{X} f^{2} \log |f| d \mu+\left(\log c_{2}\right)\|f\|_{2}^{2} & \leq s c_{1} \int_{X} f^{2} \Lambda_{+}\left(\log \left(c_{2}^{2} f^{2}\right)\right) d \mu+M_{3}\left(c_{1} s\right)\|f\|_{2}^{2} \\
& \leq s \mathcal{E}(f)+M_{3}\left(c_{1} s\right)\|f\|_{2}^{2}
\end{aligned}
$$

The last inequality is obtain from the assumption (1.10). Thus we easily conclude (1.8) with $M_{1}(s)=M_{3}\left(c_{3} s\right)+c_{4}, s>0$, and $c_{3}=c_{1}, c_{4}=-\log c_{2}$. 


\subsection{Remarks and comments.}

2.4.1. A remark about the cut-off method. For the proof of Orlicz-Sobolev type inequalities of Theorem 1.1, we have applied the cut-off method with $f_{k}$ i.e. $f_{\rho, k}$ with $\rho=2$. A similar proof of the Orlicz-Sobolev type inequality (1.10) under the assumption of Nash type inequality (1.9) can be performed with $f_{\rho, k}$ of Lemma 2.3 for any $\rho>1$. In that case, if we apply successively the circle of implications (1.8) with $M_{1} \Longrightarrow(1.9) \Longrightarrow(1.10) \Longrightarrow(1.8)$ with $\tilde{M}_{1}$. For any $\rho>1$, we have the relation

$$
\tilde{M}_{1}(t)=M_{1}\left(t(\rho-1)^{2} \rho^{-4}\right)+\log \left(\rho^{3}(\rho-1)^{-1}\right) .
$$

In terms of equivalence of these functional inequalities, it is of interest to obtain $\tilde{M}_{1}$ as close as possible to $M_{1}$, up to the additive constant $\log \left(\rho^{3}(\rho-1)^{-1}\right)$. Since in applications we can always assume that $M_{1}$ is a non-increasing function, we need to optimize the choice of $\rho>1$ of the expression $M_{1}\left(t(\rho-1)^{2} \rho^{-4}\right)$ for any $t>0$ i.e. to optimize the function $\rho \mapsto(\rho-1)^{2} \rho^{-4}$ over $(1,+\infty)$. This function attains exactly its supremum at $\rho=2$. This justifies our choice of $\rho=2$ for the proof in Section 2.2.

2.4.2. Comments. After the submission of this paper, it was drawn to our attention that the relationship between the statement (1) for a fixed $t$ and a closed version of (2), namely (2.4) for a fixed $t$, of our main Theorem 1.1 is implicitly described in Proposition 5.1.8, p.241, of the very recent book [BGL]. This is proved under the stronger assumptions that the Dirichlet form $\mathcal{E}$ is given by a carré du champ, satisfies the diffusion property, is ergodic and the measure is invariant for the associated semigroup. These conditions are imposed by the use of Proposition 3.1.17 in [BGL]. Note that, different from Proposition 3.1.17 of [BGL], Lemma 2.3 is valid for all Dirichlet forms without additional assumptions.

We also refer the reader to [BGL] for an introduction to the subject of functional inequalities treated here (in particular, Chapters 5 and 7) and also for related topics, in particular, for the measure-capacity formulations of Nash type inequalities which are not considered in this paper, see [BGL, Chap. 8]. Similarly, we can consult the book by F-Y Wang [W2].

\section{EXAMPLES OF CLASSES OF ULTRACONTRACTIVITY}

In this section, we consider three special classes of ultracontractivity. For the first two classes, we state the equivalence between the specific bound of ultracontractivity and the corresponding functional inequalities. For the 
third class, ultracontractivity is a stronger property than the pertaining functional inequalities.

3.1. Polynomial class of ultracontractivity. We say that a semigroup $\left(T_{t}\right)_{t>0}$ with generator $A$ belongs to the polynomial class of ultracontractivity of order $\nu>0$ if $c(t)=c_{1} t^{-\nu / 4}$ in (1.3) with $c_{1}>0$. This is the most common case of applications, see for instance $[\mathrm{N}],[\mathrm{CKS}],[\mathrm{VSC}],[\mathrm{G}],[\mathrm{BGL}]$. We recall that $\mathcal{E}(f)=(\sqrt{A} f, \sqrt{A} f)$ with $f \in \mathcal{D}(\sqrt{A})$. The following statements are equivalent.

(1) The semigroup $\left(T_{t}\right)_{t>0}$ belongs to the polynomial class of ultracontractivity of order $\nu$.

(2) The generator $A$ satisfies the following Nash inequality

$$
\|f\|_{2}^{2+\frac{4}{\nu}} \leq c_{2} \mathcal{E}(f)\|f\|_{1}^{\frac{4}{\nu}} .
$$

(3) The generator $A$ satisfies the following super log Sobolev inequality

$$
\int_{X} f^{2} \log \left(f /\|f\|_{2}\right) d \mu \leq t \mathcal{E}(f)+\log \left(c_{3} t^{-\frac{\nu}{4}}\right)\|f\|_{2}^{2}, \quad t>0 .
$$

(4) The generator $A$ satisfies the following Orlicz-Sobolev type inequality

$$
\int_{X} f^{2+\frac{4}{\nu}} d \mu \leq c_{4} \mathcal{E}(f)\|f\|_{2}^{\frac{4}{\nu}} .
$$

(5) The generator $A$ satisfies the following Sobolev inequality $(\nu>2)$

$$
\|f\|_{\frac{2 \nu}{\nu-2}}^{2} \leq c_{5} \mathcal{E}(f) \text {. }
$$

(6) The generator $A$ satisfies the following super Poincaré inequality

$$
\|f\|_{2}^{2} \leq t \mathcal{E}(f)+c_{6} t^{-\nu / 2}\|f\|_{1}^{2}, \quad t>0
$$

for some positive constants $c_{i}, i=1, \cdots, 6$.

The equivalences between polynomial ultracontractivity, Nash inequality, super log Sobolev inequality and Orlicz-Sobolev type inequality are deduced from (1.6), (1.7), (1.11) and Theorem 1.1. Nash inequality is super Poincaré inequality optimized over $t>0$. Historically, the first proof of equivalence between ultracontractivity and Sobolev inequality can be found in [V] and the one between ultracontractivity and Nash inequality (and implicitly super Poincaré inequality) in $[\mathrm{CKS}]$ inspired by $[\mathrm{N}]$. A direct proof of the fact that Nash inequality implies Sobolev inequality using the cut-off method can be found in [BCLS]. The Orlicz-Sobolev inequality (4) is also called Moser's inequality. 
3.2. One-exponential class of ultracontractivity. We say that a semigroup $\left(T_{t}\right)_{t>0}$ with generator $A$ belongs to the one-exponential class of ultracontractivity of order $\alpha>0$ if $c(t)=\exp \left(c_{1} t^{-\alpha}\right)$ in (1.3) with $c_{1}>0$. The following statements are equivalent.

(1) The semigroup $\left(T_{t}\right)_{t>0}$ belongs to the one-exponential class of ultracontractivity of order $\alpha$.

(2) The generator $A$ satisfies Nash type inequality (1.9) with

$$
\Lambda(\log x)=c_{2}\left[\log _{+}\left(c_{3} x\right)\right]^{1+\frac{1}{\alpha}} .
$$

(3) The generator $A$ satisfies super log Sobolev inequality (1.7) with $\beta(t)=c_{4} t^{-\alpha}$.

(4) The generator $A$ satisfies Orlicz-Sobolev type inequality (1.10) with $\Lambda$ as in (3.1).

Here $c_{i}$ are positive constants for $i=1, \cdots, 4$.

The equivalences between one-exponential ultracontractivity, Nash type inequality, super log Sobolev inequality and Orlicz-Sobolev type inequality are deduced from (1.6), (1.7), (1.11) and Theorem 1.1.

These results apply to the family of examples of Section 4.1 described below.

Comments

First, we notice the following fact. The usual semigroup proof of super Poincaré inequality under the following ultracontractivity assumption

$$
\left\|T_{t} f\right\|_{2} \leq c(t)\|f\|_{1}, \quad t>0
$$

is as follows. Let $f \in \mathcal{D}(\mathcal{E}) \cap L^{1}$ and $t>0$. By symmetry and semigroup properties, we obtain

$$
\begin{aligned}
\|f\|_{2}^{2}-\left\|T_{t / 2} f\right\|_{2}^{2} & =\left(f-T_{t} f, f\right)=\int_{0}^{t}\left(A T_{s / 2} f, T_{s / 2} f\right) d s \\
\leq \int_{0}^{t}(A f, f) d s & =t \mathcal{E}(f)
\end{aligned}
$$

because $s \rightarrow\left(A T_{s / 2} f, T_{s / 2} f\right)$ is non-increasing for all $f \in \mathcal{D}(\mathcal{E})$. Hence, the following super Poincaré inequality is satisfied

$$
\|f\|_{2}^{2} \leq t \mathcal{E}(f)+\gamma(t)\|f\|_{1}^{2}, \quad t>0
$$

with $\gamma(t)=c^{2}(t / 2)$. In the case where $c(t)=\exp \left[\frac{c}{2}\left(1+(2 t)^{-1 / \delta}\right)\right]$ for some $\delta>0$, we get $\gamma(t)=\exp \left[c\left(1+t^{-1 / \delta}\right)\right]$. By [W1, Corollary 3.3], it implies that the so-called F-Sobolev inequality of index $\delta$,

$$
\int_{X} f^{2}\left[\log \left(1+f^{2}\right)\right]^{\delta} d \mu \leq c_{1} \mathcal{E}(f)+c_{2}\|f\|_{2}^{2},
$$


holds for some constants $c_{1}, c_{2}>0$.

But below we follow another route and deduce an improved F-Sobolev inequality under the same assumption of ultracontractivity by using Theorem 1.1. In particular, this will show that (3.2) and (3.3) are not necessarily sharp for the Dirichlet form $\mathcal{E}$ despite the fact that they are equivalent in a general framework [W1, Corollary 3.3]. Indeed, in Section 1 we have seen that the following super log Sobolev inequality

$$
\int_{X} f^{2} \log |f| d \mu \leq t \mathcal{E}(f)+(\log c(t))\|f\|_{2}^{2}+\|f\|_{2}^{2} \log \|f\|_{2}, \quad t>0
$$

holds true by using [D, Theorem 2.2.3]. Now by applying Theorem 1.1, we deduce that the following F-Sobolev inequality of index $\tilde{\delta}=\delta+1$,

$$
\int_{X} f^{2}\left[\log \left(1+f^{2}\right)\right]^{\delta+1} d \mu \leq c_{1} \mathcal{E}(f)+c_{2}\|f\|_{2}^{2},
$$

holds true. More precisely, we obtain (3.5) from the Orlicz-Sobolev type inequality associated to (3.1) with $\alpha=1 / \delta$ and by adding the term $c_{2}\|f\|_{2}^{2}$. Since we are considering the one-exponential class of order $1 / \delta$, the in$\operatorname{dex} \tilde{\delta}=1+\delta$ in (3.5) is now sharp by the results described above these comments. Moreover, by applying [W1, Corollary 3.3] again, we obtain an improved super Poincaré inequality (3.2) for small $t$ with rate function

$$
\tilde{\gamma}(t)=\exp \left[\tilde{c}\left(1+t^{-1 / \tilde{\delta}}\right)\right], \quad \tilde{\delta}=1+\delta, \quad t>0
$$

in place of $\gamma(t)$ since $\lim _{t \rightarrow 0} \tilde{\gamma}(t) / \gamma(t)=0$.

The discussion above reveals some weakness of what we have called the "usual" semigroup proof of super Poincaré inequality (3.2). But note that the phenomenon described above does not occur for the polynomial class studied in Section 3.1.

3.3. Double-exponential class of ultracontractivity. We say that a semigroup $\left(T_{t}\right)_{t>0}$ with generator $A$ belongs to the double-exponential class of ultracontractivity of order $\gamma>0$ if $c(t)=\exp _{2}\left(c_{1} t^{-\gamma}\right)$ in (1.3) where $\exp _{2}=\exp \circ \exp$ and $c_{1}>0$. For this class, the situation is quite different. Ultracontractivity is strictly stronger than the other functional inequalities introduced in this paper. More precisely, if the semigroup $\left(T_{t}\right)_{t>0}$ belongs to the double-exponential class of order $\gamma>0$ then it implies

(1) The generator $A$ satisfies Nash type inequality (1.9) with

$$
\Lambda(\log x)=k_{1} \log _{+}\left(k_{2} x\right)\left[\left(\log _{+}\right)_{2}\left(k_{2} x\right)\right]^{\frac{1}{\gamma}} .
$$

(2) The generator $A$ satisfies super $\log$ Sobolev inequality (1.7) with $\beta(t)=\exp \left(k_{0} t^{-\gamma}\right)$. 
(3) The generator $A$ satisfies Orlicz-Sobolev type inequality (1.10) with $\Lambda$ as in (3.6).

Here $k_{i}$ are positive constants for $i=0,1,2$.

Ultracontractivity implies Nash type inequality by (1.6) and super log Sobolev inequality by (1.7). Theorem 1.1 says that Orlicz-Sobolev type inequality is equivalent to Nash type inequality and super log Sobolev inequality. The use of (1.11) fails for the converse. We postpone the discussion of the non equivalence of one of these functional inequalities with ultracontractivity to Section 5. Note that these functional inequalities are equivalent to each other by Theorem 1.1, independently of the ultracontractivity assumption.

These results apply to the family of examples of Section 4.2 described below.

\section{EXAmples of UltraCONTRACTIVE SEMIGROUPS}

In this section, we describe briefly examples of semigroups belonging to the one and double-exponential classes of ultracontractivity for which results of Section 3 apply. The polynomial class of ultracontractivity is classical and many examples of operators can be found in the literature. So, we will not provide a detailed account for this class but indicate very briefly some examples of operators. The first example is the Laplacian on $\mathbb{R}^{n}$ and examples elaborated on this model, see [N]. Sub-Laplacians on Lie groups of polynomial growth also provide many examples, see [VSC] and reference therein, as well as Laplace-Beltrami operators on some Riemannian manifolds, see [G, p.368], Laplacians on fractals, see e.g. [K]. The list above is not exhaustive.

Here, we focus on examples in the one- and double-exponential classes. These examples are taken from [B] and concern convolution semigroups on the infinite dimensional torus $\mathbb{T}^{\infty}$. Other examples can be found for instance in [BCS, Section 8]. Note that the study of the convolution of distributions of probability measures on topological groups is an old and vast subject and goes back at least to [ST]. See also the selected open problems described in the recent paper $[\mathrm{S}]$ and references therein.

Let $X=\mathbb{T}^{\infty}$ be the product of countable many copies of the torus $\mathbb{T}$ with its ordinary product structure. The group $\mathbb{T}^{\infty}$ is an abelian compact group. We denote by 0 the neutral element and by $\mu$ the normalized Haar measure of the group. This measure is the countable product of the normalized Haar measure on $\mathbb{T}$. 
Let $\mu_{t}$ be the brownian semigroup on $\mathbb{T}$. To a sequence $\mathcal{A}=\left\{a_{k}\right\}_{k=1}^{\infty}$ of positive numbers, we associate the product measure $\mu_{t}^{\mathcal{A}}$ on $\mathbb{T}^{\infty}$ defined by

$$
\mu_{t}^{\mathcal{A}}=\otimes_{k=1}^{\infty} \mu_{a_{k} t}, \quad t>0 .
$$

This family of probability measures $\left(\mu_{t}^{\mathcal{A}}\right)_{t>0}$ defines a symmetric convolution semigroup on $\mathbb{T}^{\infty}$ denoted by $\left(T_{t}^{\mathcal{A}}\right)_{t>0}$. Ultracontractivity reads as

$$
\left\|T_{t}^{\mathcal{A}}\right\|_{1 \rightarrow \infty}=\mu_{t}^{\mathcal{A}}(0) \in(0,+\infty]
$$

where $\mu_{t}^{\mathcal{A}}(0)$ denotes the density of $\mu_{t}^{\mathcal{A}}$ (when it exists) evaluated at 0 . The infinitesimal generator $A$ of $\left(T_{t}^{\mathcal{A}}\right)_{t>0}$ acts on the cylindrical functions, i.e. functions depending on a finite number of variables, as

$$
A f=\sum_{k=1}^{\infty} a_{k} \frac{\partial^{2} f}{\partial^{2} x_{k}} .
$$

The associated counting function $\mathcal{N}^{\mathcal{A}}$ defined by

$$
\mathcal{N}^{\mathcal{A}}(x)=\sharp\left\{k \geq 1: a_{k} \leq x\right\}, \quad x>0,
$$

is of fundamental importance for the study of ultracontractivity as it can be seen by the following examples.

4.1. Examples in one-exponential class. Let $\alpha>0$. If the sequence $\left(a_{k}\right)_{k \geq 1}$ is chosen such that $\mathcal{N}^{\mathcal{A}}(x) \sim x^{\alpha}$ as $x \rightarrow+\infty$ then

$$
\log \mu_{t}^{\mathcal{A}}(0) \sim k(\alpha) t^{-\alpha} \quad \text { as } t \searrow 0,
$$

see [B, Theorem 3.18]. Hence, the semigroup $\left(T_{t}^{\mathcal{A}}\right)_{t>0}$ belongs to the oneexponential class of ultracontractivity and results of Section 3.2 hold true for such families of $\left(a_{k}\right)_{k \geq 1}$. For example, one can take $a_{k}=k^{1 / \alpha}$.

4.2. Examples in double-exponential class. Let $\gamma>0$. If the sequence $\left(a_{k}\right)_{k \geq 1}$ is chosen such that

$$
\log \mathcal{N}^{\mathcal{A}}(x) \sim x^{\gamma /(\gamma+1)} \text { as } x \rightarrow+\infty .
$$

Then

$$
\log \log \mu_{t}^{\mathcal{A}}(0) \sim c(\gamma) t^{-\gamma} \text { as } t \searrow 0
$$

see [B, Theorem 3.27]. Hence, the semigroup $\left(T_{t}^{\mathcal{A}}\right)_{t>0}$ belongs to the doubleexponential class of ultracontractivity and results of Section 3.3 hold true for such families of $\left(a_{k}\right)_{k \geq 1}$. For example, one can take $a_{k}=[\log (k+2)]^{\delta}$ with $\delta=(\gamma+1) / \gamma$. 


\subsection{A borderline case of the double-exponential class: Davies-}

Simon's counterexample. In this section, we show that ultracontractivity and super log Sobolev inequality are not equivalent properties in the double-exponential class. For that purpose, we describe the Davies-Simon's counterexample i.e. a generator of a submarkovian semigroup satisfying a super log Sobolev inequality (1.7) with $\beta(t)=\exp (c / t)$ but not ultracontractive, see [DS, Theorem 6.1 (b) and Remark 1 p.359]. Later on, a more detailed study have been provided for a family of examples including this one in $[\mathrm{KKR}],[\mathrm{BCL}]$, [BGL, Sect.7.3]. See also the comments after Proposition 7.3.1 of [BGL] on the examples treated by this proposition concerning the one- and double-exponential classes.

Let $A$ be the operator $A f=\Delta f+\nabla U . \nabla f$ defined on smooth functions $f$ on the real line $\mathbb{R}$ with $\Delta=-\frac{d^{2}}{d x^{2}}$ and let $U: \mathbb{R} \rightarrow \mathbb{R}$ be a function of class $C^{2}$. Let $\mu$ be the invariant measure $d \mu(x)=e^{-U(x)} d x$ where $d x$ denotes the Lebesgue measure on $\mathbb{R}$. The Dirichlet form associated to $A$ is given by $\mathcal{E}(f)=\int_{\mathbb{R}}|\nabla f|^{2} d \mu$. The expected counterexample corresponds to the choice $U(x)=\left(1+x^{2}\right) \log \left(1+x^{2}\right)$. In the sequel, we denote by $\|\cdot\|_{2}^{2}$ the $L^{2}$-norm with respect to the measure $\mu$.

Theorem 4.1. Let $\left(T_{t}\right)_{t>0}$ be the semigroup associated with the infinitesimal generator $A$ defined above. Then

(1) The following log Sobolev inequality holds true. For any function $f \in \mathcal{D}(\mathcal{E})$,

$$
\int_{\mathbb{R}} f^{2} \log \left(\frac{f^{2}}{\|f\|_{2}^{2}}\right) d \mu \leq t \mathcal{E}(f)+H(t)\|f\|_{2}^{2}, \quad t>0
$$

where $H(t)$ is such that there are four constants $c_{3}, c_{4}, c_{3}^{\prime}, c_{4}^{\prime}>0$

$$
c_{3} e^{c_{4} t^{-1}} \leq H(t) \leq c_{3}^{\prime} e^{c_{4}^{\prime} t^{-1}}
$$

for $t$ small enough.

(2) The Nash type inequality (1.9) and the Orlicz-Sobolev inequality (1.10) hold true with $\Lambda$ given by (3.6) where $\gamma=1$.

(3) The semigroup $\left(T_{t}\right)_{t>0}$ is not ultracontractive.

Gathering the arguments of the proof given in [DS] is rather difficult. Thus we propose a direct but different proof of super log Sobolev inequality following [BCL]. Here, we do not pretend for novelty. A simple proof of non ultracontractivity (using subsolution method for instance) is known and well detailed in [KKR, Example 5.3]. 
Proof. Here we only prove the first statement. We divide the proof into two steps. In the first step, we prove super log Sobolev inequality (4.3) with

$$
H(t)=-\frac{1}{2} \log \left(\pi e^{2} t\right)+\sup _{x \in \mathbb{R}} V_{t}(x)
$$

where $V_{t}(x)=-t\left[-\frac{1}{2} U^{\prime \prime}(x)+\frac{1}{4}\left(U^{\prime}(x)\right)^{2}\right]+U(x)=-t V(x)+U(x)$. In the second step, we provide the estimates (4.4) of $H(t)$.

Step 1. We follow the arguments of Proposition 3.1 of [BCL], see also [BGL, Prop.7.3.1]. We start from Gross' inequality which reads as

$$
\int_{\mathbb{R}} v^{2} \log \left(\frac{v^{2}}{\|\left. v\right|_{L^{2}(d \gamma)} ^{2}}\right) d \gamma \leq 2 \int_{\mathbb{R}}|\nabla v|^{2} d \gamma
$$

where $v$ is a smooth function with compact support and $d \gamma(x)=\frac{1}{\sqrt{2 \pi}} e^{-\frac{x^{2}}{2}} d x$ is the gaussian measure. We set $G(x)=\frac{1}{\sqrt{2 \pi}} e^{-\frac{x^{2}}{2}}$ and $g=v \sqrt{G}$. By integration by parts, it yields

$$
\int_{\mathbb{R}} g^{2} \log \left(\frac{g^{2}}{\|\left. g\right|_{L^{2}(d x)} ^{2}}\right) d x \leq 2 \int_{\mathbb{R}}|\nabla g|^{2} d x-\frac{1}{2} \log \left(2 \pi e^{2}\right) \int_{\mathbb{R}}|g|^{2} d x .
$$

Let $h$ be a smooth function with compact support on $\mathbb{R}$. We set $g(x)=$ $h\left(x \sqrt{2^{-1} t}\right)$ with $t>0$. The previous inequality becomes

$$
\int_{\mathbb{R}} h^{2} \log \left(\frac{h^{2}}{\|\left. h\right|_{L^{2}(d x)} ^{2}}\right) d x \leq t \int_{\mathbb{R}}|\nabla h|^{2} d x-\frac{1}{2} \log \left(\pi e^{2} t\right) \int_{\mathbb{R}}|h|^{2} d x .
$$

Now, let $f$ be a smooth function with compact support on $\mathbb{R}$. Choose $h=$ $f e^{-\frac{U}{2}}$ in the preceding inequality. Again by integration by parts, we obtain

$$
\begin{gathered}
\int_{\mathbb{R}} f^{2} \log \left(\frac{f^{2}}{\|f\|_{L^{2}(d \mu)}^{2}}\right) d \mu \leq t \int_{\mathbb{R}}|\nabla f|^{2} d \mu-\frac{1}{2} \log \left(\pi e^{2} t\right)\|f\|_{L^{2}(d \mu)}^{2} \\
+\int_{\mathbb{R}}\left(-t\left[-\frac{1}{2} U^{\prime \prime}(x)+\frac{1}{4}\left(U^{\prime}(x)\right)^{2}\right]+U(x)\right) f^{2} d \mu .
\end{gathered}
$$

This immediately implies (4.3) with $H$ given by (4.5).

Step 2. Upper bound on $H(t)$.

Let $0<t<1$. To obtain the upper bound (4.4) on $H(t)$, it is enough to find a similar upper bound on $V_{t}$ where $V_{t}(x)$ is given by

$V_{t}(x)=t\left[-x^{2} \log ^{2}\left(e\left(1+x^{2}\right)\right)+\log \left(e\left(1+x^{2}\right)\right)\right]+\frac{2 t x^{2}}{1+x^{2}}+\left(1+x^{2}\right) \log \left(1+x^{2}\right)$.

The function $V_{t}(x)$ is clearly uniformly bounded by a constant on the set $\{x \in \mathbb{R}:|x| \leq 1\}$ when $0<t<1$. By symmetry, it suffices to bound the 
supremum of $V_{t}(x)$ for $x \in[1,+\infty)$. For any $\delta>0$ and any $x \geq 1$, it is easily shown that

$$
\delta e \log ^{2}\left(e\left(1+x^{2}\right)\right)+\log \left(e\left(1+x^{2}\right)\right) \leq\left(\delta e+\frac{1}{\log (2 e)}\right) x^{2} \log ^{2}\left(e\left(1+x^{2}\right)\right) .
$$

Now, choose $\delta$ such that $0<\delta \leq \frac{1}{2 e}\left(\frac{\log 2}{1+\log 2}\right)$. It implies $\delta e+\frac{1}{1+\log 2} \leq 1-\delta e$ and

$$
\delta e \log ^{2}\left(e\left(1+x^{2}\right)\right)+\log \left(e\left(1+x^{2}\right)\right) \leq(1-\delta e) x^{2} \log ^{2}\left(e\left(1+x^{2}\right)\right) .
$$

Then we deduce

$$
-x^{2} \log ^{2}\left(e\left(1+x^{2}\right)\right)+\log \left(e\left(1+x^{2}\right)\right) \leq \delta\left[-e\left(1+x^{2}\right) \log ^{2}\left(e\left(1+x^{2}\right)\right)\right],
$$

for all $x \geq 1$. This yields

$$
V_{t}(x) \leq t \delta\left[-e\left(1+x^{2}\right) \log ^{2}\left(e\left(1+x^{2}\right)\right)\right]+2 t+e\left(1+x^{2}\right) \log \left(e\left(1+x^{2}\right)\right) .
$$

Define $W_{s}(y):=-s y \log ^{2} y+y \log y+2 t$ with $y>1$. The function $W_{s}$ attains its supremum at $y_{0}=\exp \left(\left(1-2 s+\sqrt{1+4 s^{2}}\right)(2 s)^{-1}\right)$. Since $V_{t}(x) \leq W_{s}(y)$ for any $x$ and $y$ such that $y=e\left(1+x^{2}\right)$ and $s=t \delta$, we deduce that

$$
\sup _{x \geq 1} V_{t}(x) \leq W_{s}\left(y_{0}\right) \leq \frac{e^{-1}}{2 s+\sqrt{1+4 s^{2}}} \exp \left(\frac{1}{2 s}+\frac{\sqrt{1+4 s^{2}}}{2 s}\right)+2 t .
$$

Thus for any $0<t<\frac{\sqrt{3}}{2 \delta}$, we have $\sup _{x \geq 1} V_{t}(x) \leq \frac{e^{-1}}{2 \delta t} \exp \left(\frac{3}{2 \delta t}\right)+\frac{\sqrt{3}}{\delta}$. From this inequality, we deduce the upper bound (4.4) on $H(t)$ for $t$ small enough.

\section{Lower bound on $H$.}

Let $t>0$ and $x_{0}>0$ be such that $\log \left(e\left(1+x_{0}^{2}\right)\right)=(2 t)^{-1}$. Thus for any $0<t<\frac{1}{8}$

$$
\sup _{x \in \mathbb{R}} V_{t}(x) \geq V_{t}\left(x_{0}\right)=\left(\frac{1}{4 t}-1\right) e^{\frac{1}{2 t}-1}+\frac{1}{4 t}+\frac{1}{2}+2 t\left(1-e^{1-\frac{1}{2 t}}\right) .
$$

Thus

$$
\sup _{x \in \mathbb{R}} V_{t}(x) \geq\left(\frac{1}{4 t}-1\right) e^{\frac{1}{2 t}-1} \geq \frac{1}{8 t} e^{\frac{1}{2 t}-1} \geq e^{-1} e^{\frac{1}{2 t}} .
$$

This proves the lower bound (4.4) on $H(t)$ and completes the proof of the first statement of Theorem 4.1.

\section{OpEN PROBLEMS AND CONCLUDING REMARKS}

In this section, we address questions about equivalence between ultracontractivity and the functional inequalities introduced in this paper for the double-exponential class and beyond this class. These problems deserve to be studied due to the existence of many different ultracontractivity behaviours, see for instance [BCS, Section 6 and 8].

Here is a list of questions and open problems. 
Theorems of equivalence.

(i) To the authors' knowledge, characterization of the largest class of functions $\Theta$ in Nash type inequality or $\Lambda$ in Orlicz-Sobolev inequality or $\beta$ in super log Sobolev inequality for a generator to be equivalent to ultracontractivity of the semigroup remains an open problem.

(ii) It would be of interest to describe new stable classes of ultracontractivity as in Section 3.1 and 3.2 in view of (i).

Sharpness of known theorems.

(iii) By (1.6), ultracontractivity for a semigroup with $c(t)=\exp _{2}\left(k t^{-\gamma}\right)$ in (1.3) implies a Nash type inequality for the generator with $\Theta$ of the form

$$
\Theta(x) \simeq x \log x(\log \log x)^{\frac{1}{\gamma}}, \text { for } x \text { large enough. }
$$

It would be interesting to know whether there are (Dirichlet) operators such that both ultracontractivity and Nash type inequality are sharp with $c(t)$ and $\Theta(x)$ as above, for all or some $\gamma>0$. If the answer is positive, this would show that (1.6) is sharp for the double-exponential class.

(iv) In the opposite direction, if an operator satisfies a Nash type inequality with $\Theta(x) \simeq x \log x(\log \log x)^{\frac{1}{\gamma}}$ for $x$ large enough then by (1.11) the semigroup is ultracontractive with $c(t)=\exp _{2}\left(k t^{-\alpha}\right)$ and the defective exponent $\alpha=\frac{\gamma}{1-\gamma}$ when $\gamma \in(0,1)$. It would be interesting to know whether there are (Dirichlet) operators such that both ultracontractivity and Nash type inequality are sharp with $\Theta(x)$ and $c(t)$ as above for some or all $\gamma \in(0,1)$. If the answer is positive, this would show that (1.11) is sharp for the double-exponential class.

(v) Also in another direction, if an operator satisfies a stronger Nash type inequality with $\Theta(x) \simeq x \log x(\log \log x)^{\frac{1}{\gamma}+1}$ for $x$ large enough then by (1.11) the semigroup is ultracontractive with $c(t)=\exp _{2}\left(k t^{-\gamma}\right)$. A similar question to the one of (iv) arises for some or all $\gamma>0$. If the answer is positive, this would show that (1.11) is sharp but (1.6) is not.

Concluding remarks.

At the present time, we are not able to formulate a conjecture on a theorem of general equivalence. In this regard, it will be interesting to compare Theorem 2.2.7 of [D] and Coulhon's result (1.11) in light of Theorem 1.1, see also Theorem 7.1.2 and Theorem 7.4.5 of [BGL]. Indeed, such a relation is not clear despite the fact that ultracontractivity (1.11) appears to be more direct for the double-exponential class. In that direction, the contribution of Theorem 1.1 is important since it describes the exact correspondence between super log Sobolev inequalities and Nash type inequalities. As a consequence, it should be possible to compare the ultracontractive bounds 
obtained by both methods discussed above. The authors of this paper do not know if such a comparison is really possible due to the fact that Theorem 7.1.2 of [BGL] treats also hypercontractivity and Theorem 7.4.5 of [BGL] apparently does not. A restricted open problem is to find under what general conditions on $\beta$ in super $\log$ Sobolev inequality (1.7) and $\Lambda$ in Nash type inequality (1.6) related by (1.5) lead to comparable ultracontractive bounds by applying both methods.

To conclude this paper, we conjecture that the super log Sobolev profile $H_{0}$ defined by

$$
H_{0}(t)=\sup \left(\int_{X} f^{2} \log f^{2} d \mu-t \mathcal{E}(f): f \in \mathcal{D}(\mathcal{E}),\|f\|_{2}^{2}=1\right), \quad t>0
$$

of Davies-Simon's counterexample satisfies the same lower estimate as $H$ in (4.4). Note that the upper bound holds trivially by minimality of $H_{0}$.

Acknowledgements. The authors thank the referee(s) for their comments and suggestions leading to improvements of this paper, and in particular for drawing our attention to the very recent book [BGL].

\section{REFERENCES}

[A] G. Allain, Sur la représentation des formes de Dirichlet, Ann. Inst. Fourier 25 (1975), 1-10.

[B] D. Bakry, L'hypercontractivité et son utilisation en théorie des semigroupes, in Markov semigroups at Saint-Flour. Probability at SaintFlour. Springer, Heidelberg, 2012. pp. 1-114.

[BCL] D. Bakry, D. Concordet and M. Ledoux, Optimal heat kernel bounds under logarithmic Sobolev inequalities, ESAIM Probab. Statist. 1 (1995/97), 391-407 (electronic).

[BCLS] D. Bakry, T. Coulhon, M. Ledoux and L. Saloff-Coste, Sobolev inequalities in disguise, Indiana Univ. Math. J. 44 (1995), 1033-1074.

[BGL] D. Bakry, I. Gentil and M. Ledoux, Analysis and geometry of Markov diffusion operators. Grundlehren der Mathematischen Wissenschaften, 348. Springer, 2014.

[B] A.D. Bendikov, Symmetric stable semigroups on the infinite-dimensional torus, Exposition. Math. 13 (1995), 39-79.

[BCS] A.D. Bendikov, T. Coulhon and L. Saloff-Coste, Ultracontractivity and embedding into $L^{\infty}$, Math. Ann. 337 (2007), 817-853. 
[BM] A.D. Bendikov and P. Maheux, Nash type inequalities for fractional powers of non-negative self-adjoint operators, Trans. Amer. Math. Soc. 359 (2007), 3085-3097.

[CKS] E.A. Carlen, S. Kusuoka and D.W. Stroock, Upper bounds for symmetric Markov transition functions, Ann. Inst. H. Poincaré Probab. Statist. 23 (1987), 245-287.

[C] T. Coulhon, Ultracontractivity and Nash type inequalities, J. Funct. Anal. 141 (1996), 510-539.

[D] E.B. Davies, Heat kernels and spectral theory, Cambridge Tracts in Mathematics, 92. Cambridge University Press, Cambridge, 1989.

[DS] E. B. Davies and B. Simon, Ultracontractivity and the heat kernel for Schrödinger operators and Dirichlet Laplacians, J. Funct. Anal. 59 (1984), 335-395.

[F] M. Fukushima, Dirichlet forms and Markov processes, North-Holland Mathematical Library, 23. North-Holland Publishing Co., Amsterdam-New York; Kodansha, Ltd., Tokyo, 1980.

[G] A. Grigor'yan, Heat kernel and analysis on manifolds, AMS/IP Studies in Advanced Mathematics 47, 2009.

[GH] A. Grigor'yan and J. Hu, Upper bounds of heat kernels on doubling space, Moscow Math. J. 14 (2014) 505-563.

[G1] L. Gross, Logarithmic Sobolev inequalities, Amer. J. Math. 97 (1976), 1061-1083.

[G2] L. Gross, Logarithmic Logarithmic Sobolev inequalities and contractivity properties of semigroups, Dirichlet forms (Varenna, 1992), Lecture Notes in Math., 1563, Springer, Berlin, 1993, 54-88.

[KKR] O. Kavian, G. Kerkyacharian and B. Roynette, Quelques remarques sur l'ultracontractivité, (French) [Some remarks about ultracontractivity] J. Funct. Anal. 111 (1993), 155-196.

[K] J. Kigami, Local Nash inequality and inhomogeneity of heat kernels, Proc. London Math. Soc. 89 (2004), 525-544.

[N] J. Nash, Continuity of solutions of parabolic and elliptic equations, Amer. J. Math. 80 (1958), 931-954.

[S] L. Saloff-Coste, Analysis on compact Lie groups of large dimension and on connected compact groups, Colloq. Math. 118 (2010), 183199.

[ST] V. V. Sazonov and V. N. Tutubalin, Probability distributions on topological groups, Theor. Prob. Appl. 11 (1966), 1-45. 
[V] N. Th. Varopoulos, Hardy-Littlewood theory for semigroups, J. Funct. Anal. 63 (1985), 240-260.

[VSC] N. Th. Varopoulos, L. Saloff-Coste and T. Coulhon, Analysis and geometry on groups, Cambridge Tracts in Mathematics, 100. Cambridge University Press, Cambridge, 1992.

[W1] F-Y. Wang, Functional inequalities for empty essential spectrum, J. Funct. Anal. 170 (2000), 219-245.

[W2] F-Y. Wang, Functional Inequalities, Markov Processes and Spectral Theory, Science Press, Beijing, 2004.

Dipartimento di Matematica "F. Brioschi", Politecnico di Milano, Piazza Leonardo Da Vinci 32, 20133, Milano, Italy

E-mail address: marco.biroli@polimi.it

Fédération Denis Poisson (MAPMO, UMR-CNRS 7349), Département de Mathématiques, Université d'Orléans, 45067 Orléans Cedex 2, France

E-mail address: patrick.maheux@univ-orleans.fr 